\title{
A man with spontaneously swollen eye - Orbital emphysema following forceful nose-blowing.
}

so eun $\mathrm{kim}^{1}$

${ }^{1}$ Jeonbuk National University Hospital

June 1,2020

\begin{abstract}
A 33-year-old male admitted with sudden and spontaneous painless left infraorbital swellings after forceful nose-blowing. He was diagnosed orbital emphysema with orbital floor blow-out fracture. This case shows that forceful nose-blowing can cause orbital floor fracture and subcutaneous orbital emphysema by rapid increase in pressure in the upper airways.
\end{abstract}

\section{Orbital emphysema with orbital floor blow-out fracture.}

He was treated conservatively and discharged with explanation of it might expected to resolve spontaneously. He was instructed to avoid blowing his nose and any other activities that may lead to increased pressure in the nasal cavity, including diving or flying.

\section{Discussion}

Cases of spontaneous orbital emphysema caused by non-traumatic reasons are very rare. ${ }^{1}$ Rapid increase in pressure in the upper airways during sneezing, coughing, nose blowing is related to barotrauma which very rarely leads to orbital wall fracture. The pressure evoked by forceful nose blowing rises to $66 \mathrm{mmHg}$, as estimated. Sneezing with the oral and nasal passages blocked may evoke a pressure up to $176 \mathrm{mmHg}{ }^{2}$ Orbital emphysema without complication usually resolves spontaneously within 2 weeks.

\section{Reference}

1. Myers S, Bell D. Orbital blowout fracture from nose blowing.BMJ Case Reports. 2018;2018:bcr-2018224633 .

2. Rzymska-Grala I, Palczewski P, Błaż M, Zmorzyński M, Gołębiowski M, Wanyura H. A peculiar blow-out fracture of the inferior orbital wall complicated by extensive subcutaneous emphysema: A case report and review of the literature. Polish journal of radiology.2012;77:64.

\section{Legend}

Figure 1. Non-tender left periorbital swelling.

Figure 2. A fundoscopy revealed no abnormal findings in the vitreous body or the retina.

Figure 3. Computed tomography (CT) shows subcutaneous emphysema around left orbit. (A) Axial CT shows fracture of the left inferior orbital wall fracture (white arrows), herniation of orbital fat through the fracture (black arrows), and orbital emphysema (arrowheads). (B) Coronally CT shows fracture of the left inferior orbital wall fracture (white arrows), herniation of orbital fat through the fracture (black arrows), and orbital emphysema (arrowheads). (C) Sagittal CT shows fracture of the left inferior orbital wall fracture (white arrows), and orbital emphysema (arrowheads). 


\section{Key word}

Orbit, periorbital emphysema, orbital floor fracture, sneezing.

\section{Key Clinical Message}

Rapid increase in pressure in the upper airways during sneezing, coughing, nose blowing is related to barotrauma which can cause orbital wall fracture. This case shows that forceful nose-blowing can cause orbital floor fracture and subcutaneous orbital emphysema.

\section{Conflict of interest}

There is no conflict of interest to declare.

\section{Author Contributions}

The author solely contributed toward the writing of this manuscript.

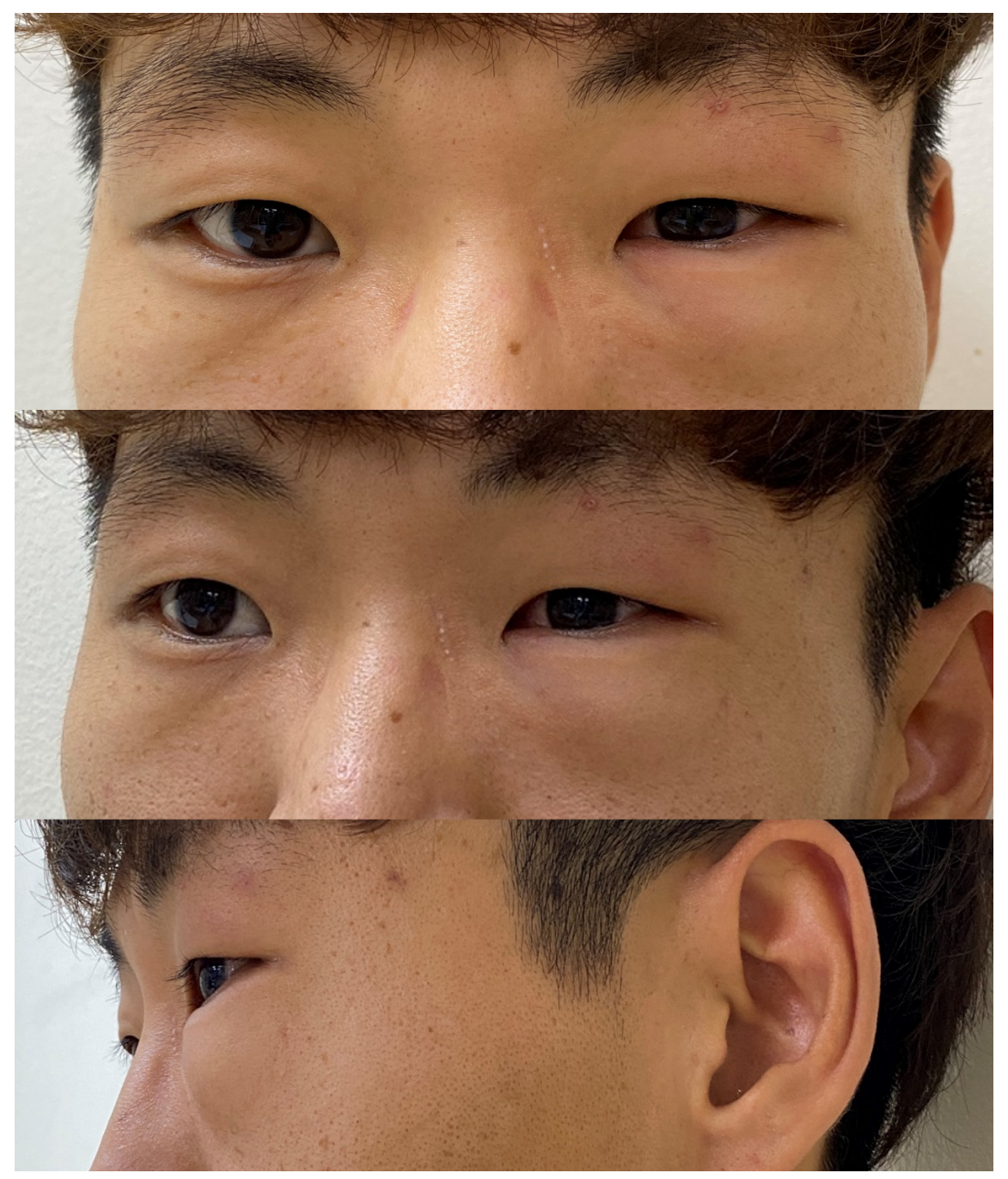



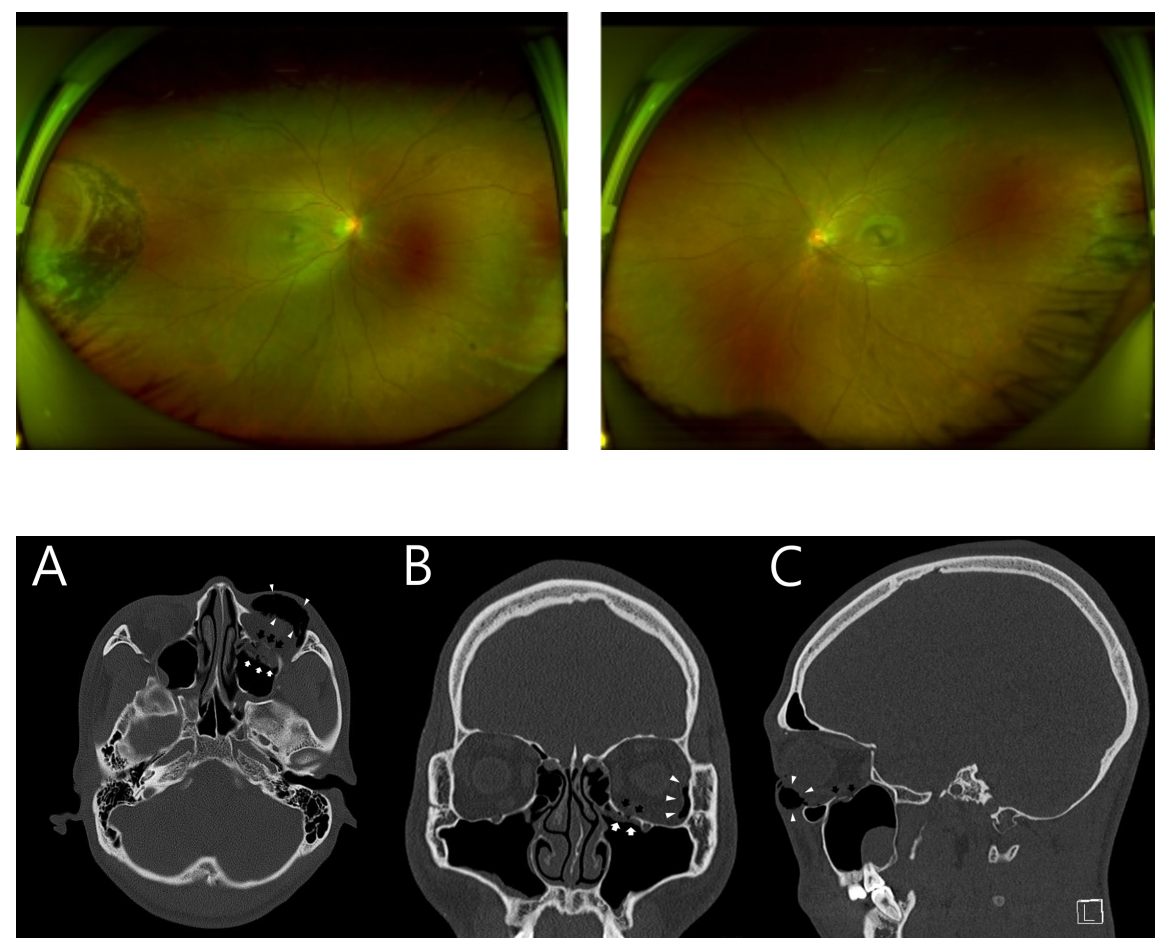